\title{
Is the pleural fluid transudate or exudate? A revisit of the diagnostic criteria
}

\author{
J Joseph, P Badrinath, G S Basran, S A Sahn
}

\begin{abstract}
Background-Pleural effusions are classified into transudates and exudates based on criteria developed in the 1970s. However, their accuracy has not been evaluated. We compared the performance of the pleural fluid absolute lactic dehydrogenase level (FLDH), fluid to serum ratio of LDH (LDHR), and fluid to serum ratio of total protein (TPR). TPR has been used instead of the absolute value of fluid protein based on the observation that fluid protein is influenced by changes in the serum protein concentration. However, the rationale for using LDHR remains unexplored.
\end{abstract}

Methods-Of 212 consecutive patients with pleural effusions, four with multiple causes and eight with an uncertain diagnosis were excluded. ROC curves were generated using sensitivity and 1-specificity values for TPR, FLDH, and LDHR and positive likelihood ratios (LR +ve) were computed using the optimum cut off values. The correlation between pleural fluid and serum concentrations of total protein and LDH was also estimated. Results-Of 200 effusions studied, 156 were exudates and 44 were transudates. The optimum cut off levels were: FLDH 163 IU/1, TPR 0.5, LDHR 0.6, and the FLDH-TPR combination 163 and 0.4 , respectively. The area under the curve (AUC) with $95 \%$ confidence interval (CI) was: 0.89 (0.86 to 0.96 ) for FLDH, 0.86 (0.80 to 0.91$)$ for TPR, $0.82(0.77$ to 0.89$)$ for LDHR, and $0.90(0.86$ to 95$)$ for FLDH-TPR. A significant correlation was observed between serum and pleural fluid protein levels in transudates and exudates $(r=0.5$ and 0.6 , respectively), but the correlation between serum and pleural fluid LDH levels was insignificant.

Conclusion-FLDH is the most accurate marker for the diagnostic separation of transudates and exudates and LDHR has no role in this process. Combining TPR with FLDH appears to improve the diagnostic accuracy slightly.

(Thorax 2001;56:867-870)

Keywords: exudates; transudates; pleural effusions

Correspondence to: Dr J Joseph

jioseph@uaeu.ac.ae

Received 8 November 2000 Returned to authors 26 March 2001

Revised version received 26 April 2001

Accepted for publication

28 June 2001 absolute lactic dehydrogenase (FLDH) level is $>200 \mathrm{IU} / 1$, or the pleural fluid to serum ratio of $\mathrm{LDH}$ (LDHR) is $>0.6$, and transudates if the TPR is $<0.5$, FLDH level is $<200 \mathrm{IU} / 1$, and the LDHR is $<0.6 .^{3}$

The accuracy of these tests has been analysed using conventional histograms and was reported to approach $100 \% .{ }^{4}$ However, these analyses were done using a single sensitivity and specificity derived from a scatter plot. Calculation of the accuracy based on a single sensitivity/specificity pair provides only a brief glimpse of the performance of a test and does not reveal its true diagnostic value. ${ }^{5}$ Furthermore, in the diagnostic separation of pleural effusions into transudates and exudates, the performance of these tests was not compared individually or in combination. We have therefore analysed the global performance of these tests using receiver operator characteristics (ROC) analysis in a heterogeneous group of patients with a known cause for their pleural effusion. The TPR value was chosen instead of the absolute value of fluid total protein to differentiate the pleural effusions, based on the observation that the total protein concentration in the pleural fluid is influenced by changes in the protein concentration in the serum. ${ }^{3}$ However, the scientific basis for using LDHR has not been explored by previous investigators. ${ }^{3}$

\section{Methods}

Patients referred to the respiratory unit at Rotherham General Hospital, UK for a prospective investigation of the dynamics of pleural effusion formation and removal from January 1989 to June 1991 were included in the study. ${ }^{6}$ The cause of the pleural effusion was determined using established clinical criteria. ${ }^{3}$ All patients were followed for at least 3 months or until a final cause of the pleural effusion was established. Blood and pleural effusion samples collected and stored were later analysed for glucose, albumin, total protein, and $\mathrm{LDH}$. $\mathrm{LDH}$ was measured with a Boehringer Mannheim kit according to established methods and the results were expressed in IU/1. ${ }^{7}$ The upper limit for the normal serum $\mathrm{LDH}$ level in our laboratory during the study was $200 \mathrm{IU} / 1$.

During the study period pleural effusion samples from 212 patients were collected. However, eight patients with an uncertain diagnosis and four with possible multiple causes for the pleural effusion were excluded from the analysis, leaving 200 (94\%) for analysis. These 200 effusions were classified into exudates or transudates according to the clinical criteria established by Light and colleagues. $^{3}$ 
Table 1 Causes of pleural effusions

\begin{tabular}{lc}
\hline Diagnosis & $n(\%)$ \\
\hline Exudates (n=156) & $47(23.5 \%)$ \\
Lung cancer & $34(17 \%)$ \\
Metastatic cancer & $9(4.5 \%)$ \\
Mesothelioma & $20(10 \%)$ \\
Parapneumonic & $14(7 \%)$ \\
Empyema & $3(1.5 \%)$ \\
Tuberculosis & $11(5.5 \%)$ \\
Pulmonary embolism & $6(3 \%)$ \\
Pancreatitis & $5(2.5 \%)$ \\
Connective tissue disease & $7(3.5 \%)$ \\
Other exudates & \\
Transudates (n=44) & $37(18.5 \%)$ \\
Cardiac failure & $5(2.5 \%)$ \\
Cirrhosis of liver & $2(1 \%)$ \\
Nephrotic syndrome & 200 \\
Total & \\
\hline
\end{tabular}

^Dressler's syndrome 2 , post-thoracic surgery 3, lymphangioleiomatosis 1 , subphrenic abscess 1 .

\section{STATISTICAL ANALYSIS}

To compare the performance of TPR, FLDH, LDHR, and a combination of TPR and FLDH in the diagnostic separation of transudates and exudates, ROC curves were generated for each of the criteria by plotting the sensitivity against 1-specificity using the Stats Direct statistical package. This program generates ROC curves using continuous data points. The area under the curve (AUC) with 95\% confidence intervals was calculated by the software using methods described by Hanley and $\mathrm{McNeil} .{ }^{8}$ Optimum cut off points for FLDH, TPR, LDHR, and the FLDH-TPR combination were established by selecting the points of test values that provided the greatest sum of sensitivity and specificity - that is, the point closest to the top left hand corner on the ROC curve. In order to obtain a ROC plot for a combination of criteria to diagnose transudates, the optimum cut off point from the best ROC curve was selected as the baseline criterion and varying test values of the second best criterion were added to this by ranking of the values the second best criteria; this AND logic has been used previously by Light et al. ${ }^{3}$

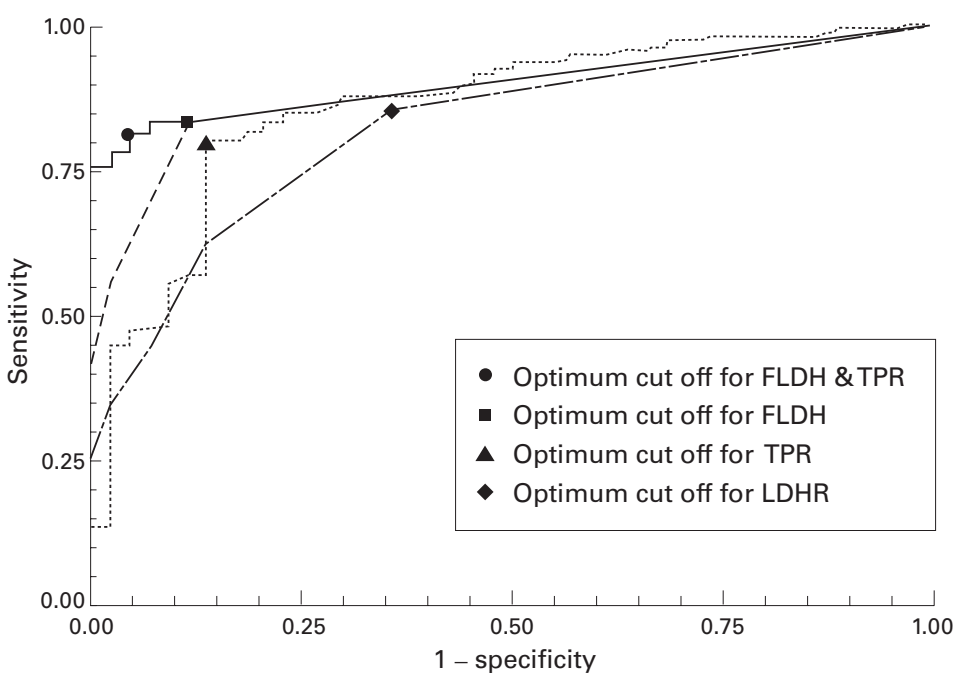

Figure 1 ROC plots of pleural fluid values of FLDH, TPR, LDHR and FLDH-TPR combination. The optimum cut off level was determined by selecting points of test values that provided the greatest sum of sensitivity and specificity. The optimum cut off levels for FLDH, TPR, and LDHR were $163 \mathrm{IU} / l, 0.5$, and 0.6, respectively, and $163 \mathrm{IU} / \mathrm{l}$ and 0.4 for the FLDH-TPR combination.
Table 2 Area under the curve (AUC) for the various test criteria (Wilcoxon estimate)

\begin{tabular}{lll}
\hline Criteria & AUC & 95\% CI \\
\hline FLDH & 0.89 & 0.86 to 0.96 \\
TPR & 0.86 & 0.80 to 0.91 \\
LDHR & 0.82 & 0.77 to 0.89 \\
FLDH + TPR & 0.90 & 0.86 to 0.95
\end{tabular}

FLDH $=$ pleural fluid absolute lactic dehydrogenase; TPR $=$ pleural fluid to serum ratio of total protein; LDHR = pleural fluid to serum ratio of $\mathrm{LDH}$.

Positive likelihood ratios (LR+ve) were calculated for the optimum cut off points of FLDH, TPR, LDHR, and the FLDH-TPR combination. $\mathrm{LR}+\mathrm{ve}$ is the likelihood that the test would be positive in a patient with disease compared with the likelihood that the same result would be expected in a patient without the disease. The higher the LR, the better is the test in discriminating between those with and without disease. Finally, the correlation between serum and pleural fluid concentrations of total protein and $\mathrm{LDH}$ was estimated using Pearson's correlation coefficient.

\section{Results}

Of the 200 patients with a pleural effusion, 116 were men and 84 were women. The mean (SE) age was 62 (1.1) years, median 63 years (range 14-94). Table 1 shows the cause of the pleural effusions in the study patients. Forty four $(22 \%)$ were transudates and the remaining 156 $(78 \%)$ were exudates. Congestive heart failure was the most common cause for a transudative pleural effusion and represented 19\% (37/200) of the total. Malignancy was the cause in 90 of $200(45 \%)$, and infection in 37 of 200 (19\%). As expected, the mean test concentrations of TPR, FLDH, and LDHR were significantly different in transudates and exudates (all $\mathrm{p}<0.0001)$.

The ROC plots for FLDH, TPR, LDHR, and FLDH-TPR are shown in fig 1 . At all test values FLDH was more sensitive and specific than TPR and LDHR. The optimum cut off levels for FLDH, TPR, and LDHR were $163 \mathrm{IU} / 1,0.5$, and 0.6, respectively, and $163 \mathrm{IU} / 1$ and 0.4 for the FLDH-TPR combination. Table 2 shows the AUC with 95\% CI for the three criteria and for the FLDH-TPR combination. FLDH was the best of the three tests, although there was minimal overlapping of confidence intervals. The AUC for the FLDH-TPR combination was marginally better than the AUC for FLDH alone, but the difference was not statistically significant. The addition of different test values of LDHR to the FLDH-TPR combination did not improve the accuracy.

The false negative and false positive rates at the optimum cut off values were higher for TPR $(19.8 \%$ and $16.6 \%)$ than for FLDH $(13.6 \%$ and $11.3 \%)$. FLDH was more accurate $(84.5 \%)$ than TPR $(81.5 \%)$ in differentiating transudates from exudates. FLDH correctly identified five more patients with an exudative effusion than TPR. Furthermore, FLDH correctly identified one more patient as having a transudate than TPR. Table 3 lists the positive likelihood ratios (LR +ve) with 95\% 
Table 3 Positive likelihood ratios ( $L R+v e)$ at optimum cut off points

\begin{tabular}{lll}
\hline Test & LR+ve & $95 \%$ CI \\
\hline FLDH + TPR & 18 & 5.4 to 65 \\
FLDH & 7.3 & 3.5 to 17 \\
TPR & 5.9 & 3 to 12.6 \\
LDHR & 2.4 & 1.7 to 3.6 \\
\hline
\end{tabular}

FLDH $=$ pleural fluid absolute lactic dehydrogenase; TPR = pleural fluid to serum ratio of total protein; LDHR = pleural fluid to serum ratio of $\mathrm{LDH}$.

CI for the four tests. A combination of FLDH and TPR had the highest LR followed by FLDH, TPR, and LDHR, although the confidence interval was wider.

Figure 2 shows the correlation between serum and pleural fluid concentrations of total protein and $\mathrm{LDH}$ in transudates and exudates. A significant correlation was seen between serum and pleural fluid total protein concentrations in transudates and exudates $(r=0.5$ and
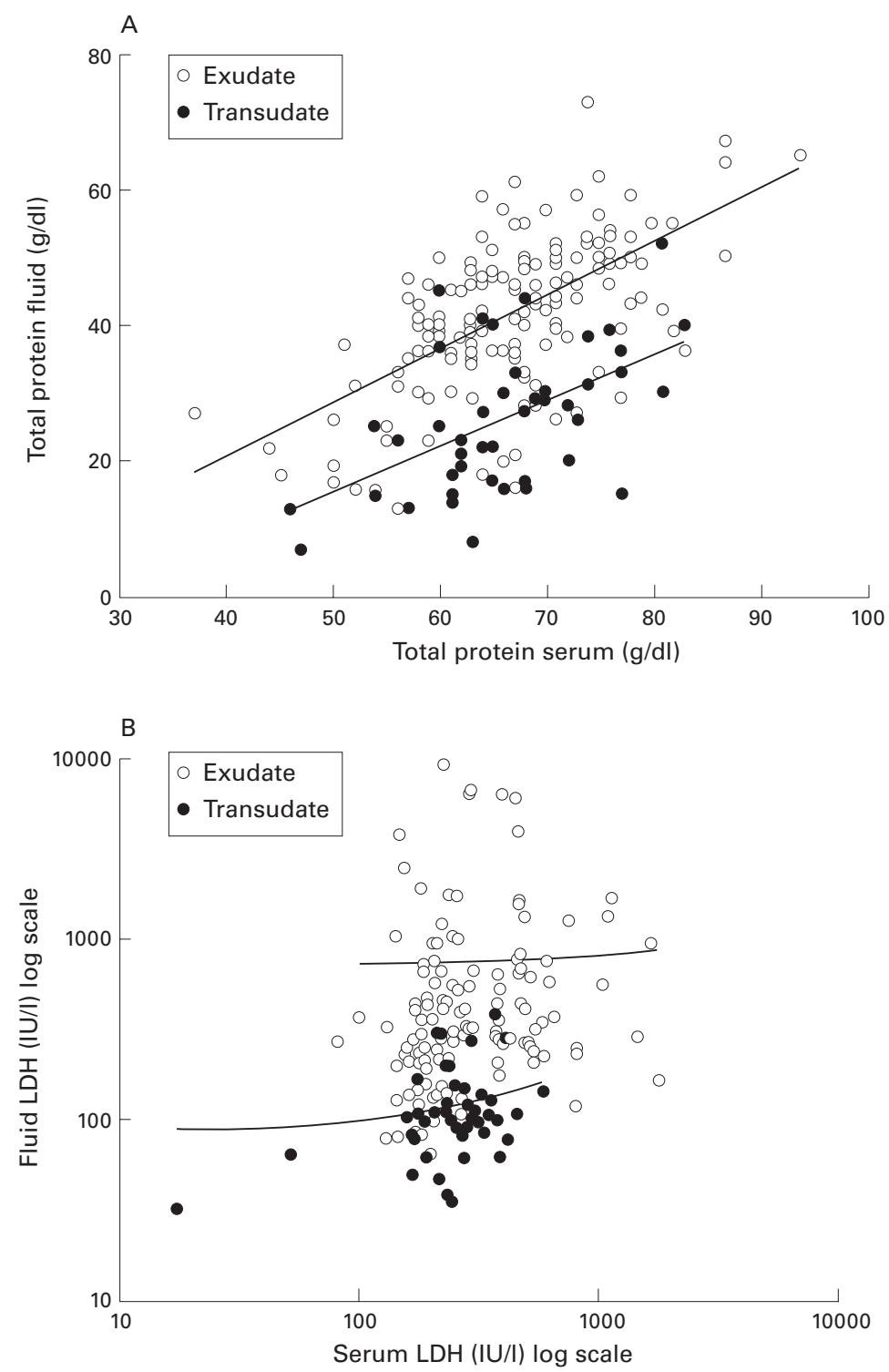

Figure 2 (A) Correlation between pleural fluid and serum total protein in transudates and exudates $(r=0.5$ and $r=0.6$, respectively, both $p<0.001)$ suggesting that the pleural fluid total protein value is influenced by the serum value. (B) Serum to fluid LDH plot showing no significant correlation in transudates or exudates $(r=0.02$ and $r=0.2$, respectively), suggesting that the pleural fluid LDH concentration is independent of the serum LDH concentration.
0.6 , respectively, $\mathrm{p}<0.01$ ), but there was no significant correlation between serum and pleural fluid concentrations of LDH in transudates or exudates $(r=0.02$ and 0.2 , respectively, $\mathrm{p}>0.05)$.

\section{Discussion}

Diagnostic separation of pleural effusions into transudates and exudates is a useful strategy for evaluating patients with pleural effusions. A transudate indicates limited diagnostic possibilities and generally precludes further diagnostic testing. The causes of the pleural effusions in our subjects were similar to those of Light and colleagues. ${ }^{3}$ Our analysis indicates that the pleural fluid absolute LDH level is the most accurate test for the diagnostic separation of pleural effusions into transudates and exudates. The optimum cut off levels in our study for TPR (0.5) and LDHR (0.6) were similar to that of Light and colleagues. However, the FLDH value of $163 \mathrm{IU} / \mathrm{ml}$ in our study was different. The upper limit of the serum LDH level in our study was $200 \mathrm{IU} / \mathrm{ml}$ and the optimal cut off point of $163 \mathrm{IU} / \mathrm{ml}$ is $82 \%$ of this value. Compared with this, the optimum cut off point of $200 \mathrm{IU} / \mathrm{ml}$ in the study by Light et al was $66 \%$ of their upper limit for the serum LDH level (300 IU/ml). We therefore suggest that, whatever method is used for estimating the FLDH level, $82 \%$ of the upper limit of the normal serum LDH level should be used to differentiate between exudates and transudates.

FLDH has previously been shown to be better than TPR in the diagnostic differentiation of pleural effusions. ${ }^{9}$ This superior performance may be attributed to the increased local generation of FLDH in the pleural cavity in all exudative processes and the relatively low level of generation in transudates. The increase in the local concentration of $\mathrm{LDH}$ has been documented in several disease processes such as pulmonary infarction and malignancy, ${ }^{10}$ and also in pleural inflammatory conditions. ${ }^{11}$ Furthermore, activated, injured or dead mesothelial cells and other inflammatory cells that have migrated into the pleural space in inflammatory processes are an important source of pleural fluid LDH. ${ }^{12}{ }^{13}$ An increasing LDH concentration in the pleural fluid is therefore a sensitive marker of an underlying exudative process. A local source for increased $\mathrm{LDH}$ generation in transudative pleural effusions has not been reported.

In the diagnostic separation of pleural effusions the TPR has been chosen instead of the absolute value of total protein based on the assumption that the concentration of total protein in the pleural fluid is influenced by changes in serum protein levels; this is confirmed by our data showing a significant correlation between serum and pleural fluid total protein concentrations. If this is true for total protein, a similar relationship might also apply to $\mathrm{LDH}$ dynamics. However, our analysis has clearly demonstrated that there was no significant correlation between the serum and pleural fluid concentrations of $\mathrm{LDH}$ in transudates or exudates (fig 2B), so it is reasonable to suggest 
that the serum concentration of $\mathrm{LDH}$ does not influence the pleural fluid concentration. It therefore follows that there is no basis for using the $\mathrm{LDH}$ ratio in the diagnostic separation of pleural effusions, unlike the total protein ratio where the pleural fluid concentration is influenced by the serum concentration. On the contrary, as the pleural fluid concentration of $\mathrm{LDH}$ is independent of the serum concentration, the LDH concentration in any fluid with a relatively low serum $\mathrm{LDH}$ concentration can result in a high LDHR causing false classification of a transudate as an exudate.

The lack of reliability of TPR as a diagnostic measure has been documented previously. ${ }^{14}$ This may be related to the dynamics of protein movement from plasma to the pleural space in cardiac failure. The degree of pulmonary venous hypertension is a major determinant of pleural effusion formation in heart failure. ${ }^{16}{ }^{17}$ In addition, the concentration of total protein leaking out of the microcirculation is proportional to the degree of elevation of pulmonary capillary wedge pressure. ${ }^{18}$ Furthermore, in transudative ascites secondary to portal hypertension, a process similar to congestive heart failure, the serum protein concentration and the degree of portal pressure determine the ascitic fluid total protein concentration. ${ }^{19}$ Thus, in congestive heart failure, as in portal hypertension, the total protein concentration in pleural fluid could be a reflection of the degree of pulmonary venous hypertension causing the pleural effusion. This may partly be the basis for the so called pseudoexudate reported by Chakko and coworkers ${ }^{15}$ in congestive heart failure.

If FLDH rather than TPR had been used as the sole diagnostic test in our series of 200 patients, one fewer patient would have been subjected to further diagnostic workup. On the other hand, FLDH also correctly identified five more exudative effusions. The cost effectiveness of using FLDH as a diagnostic test therefore merits attention, although this was not the main emphasis of our current investigation. In addition, we have shown that LDHR has no diagnostic usefulness and measurement of serum $\mathrm{LDH}$ concentrations can therefore be avoided, leading to potential cost savings.

In conclusion, FLDH is the most accurate test in the diagnostic separation of transudates and exudates. The value of FLDH may differ depending on the methodology used for estimation. ${ }^{7}$ Whatever the method used for the estimation of $\mathrm{LDH}$, our data indicate that $82 \%$ of the upper limit of the normal serum value should correspond to the optimum cut off value for pleural fluid levels of $\mathrm{LDH}$. Combining TPR and FLDH is meaningful as the combination improves the test accuracy and the likelihood ratio. As the pleural fluid concentration of $\mathrm{LDH}$ is not influenced by the serum concentration, there is no basis for using LDHR in the diagnostic separation of pleural effusions. We therefore propose that the diagnostic separation of pleural effusions into transudates and exudates should be based only on TPR and FLDH levels. If confirmed by other investigators, this will have an impact on the diagnostic separation of pleural effusions into transudates and exudates.

The authors would like to thank Mr Steve Viney for carrying out the pleural fluid biochemical analyses.

1 Black LF. The pleural space and pleural fluid. Mayo Clin Proc 1972;47:493-506.

2 Sahn SA. State of the art. The pleura. Am Rev Respir Dis 1988;138:184-234.

3 Light RW, Macgregor MI, Luchsinger PC, et al. Pleural effusions: the diagnostic separation of transudates and exudates. Ann Intern Med 1972;77:507-13.

dates. Ann Intern Med 1972;77:507-13.
4 Jay SJ. Diagnostic procedures for pleural disease. Clin Chest Med 1985;6:33-48.

5 Heffner JE, Brown LK, Barbieri CA. Diagnostic value of tests that discriminate between exudative and transudative pleural effusions. Primary Study Investigators. Chest 1997; 11:970-80.

6 Smith MJ, Joseph J, Flatman WD, et al. A dual-isotope method for studying protein kinetics in pleural effusions in humans. Nucl Med Commun 1992;13:432-9.

7 Scandinavian Society for Clinical Chemistry and Clinical Physiology. Recommended methods for the determination of four enzymes in blood. Scand F Clin Lab Invest 1994;33: 291-5.

8 Hanley JA, McNeil BJ. A method of comparing the areas under receiver operating characteristic curves derived from the same cases. Radiology 1983;148:839-43.

9 Chandrasekhar AJ, Palatao A, Dubin A, et al. Pleural fluid lactic acid dehydrogenase activity and protein content. Value in diagnosis. Arch Intern Med 1969;123:48-50.

10 Whitaker D, Papadimitriou JM, Walters MN. The Whitaker D, Papadimitriou JM, Walters MN. The
mesothelium: a histochemical study of resting mesothelial mesothelium: a histochemical stu
cells. F Pathol 1980;132:273-84.

11 Vergnon JM, Guidollet J, Gateau O, et al. Lactic dehydrogenase isoenzyme electrophoretic patterns in the diagnosis of pleural effusion. Cancer 1984;54:507-11.

12 Paavonen T, Liippo K, Aronen $\mathrm{H}$, et al. Lactate dehydrogenase, creatine kinase, and their isoenzymes in pleural effusions. Clin Chem 1991;37:1909-12.

13 Whitaker D, Papadimitriou JM, Walters MN. The mesothelium: a cytochemical study of "activated" mesothelial cells. $\mathcal{F}$ Pathol 1982;136:169-79.

14 Melsom RD. Diagnostic reliability of pleural fluid protein estimation. $\mathcal{f} R$ Soc Med 1979;72:823-5.

15 Chakko SC, Caldwell SH, Sforza PP. Treatment of congestive heart failure. Its effect on pleural fluid chemistry. Chest 1989;95:798-802.

16 Allen S, Gabel J, Drake R. Left atrial hypertension causes pleural effusion formation in unanesthetized sheep. Am $\mathcal{F}$ Physiol 1989;257:H690-2.

17 Wiener-Kronish JP, Matthay MA, Callen PW, et al. Relationship of pleural effusions to pulmonary hemodynamics in patients with congestive heart failure. Am Rev Respir Dis 1985;132:1253-6.

18 Broaddus VC, Wiener-Kronish JP, Staub NC. Clearance of lung edema into the pleural space of volume-loaded anesthetized sheep. F Appl Physiol 1990;68:2623-30.

19 Hoefs JC. Serum protein concentration and portal pressure determine the ascitic fluid protein concentration in patients with chronic liver disease. F Lab Clin Med 1983;102:260- 